\title{
Unique Pediatric Gallstones Composed of Calcium Oxalate Phosphate
}

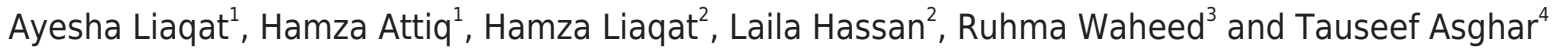 \\ ${ }^{1}$ Department of Surgery, Services Institute of Medical Sciences, Lahore, Pakistan \\ ${ }^{2}$ Department of Surgery, Khawaja Muhammad Safdar Medical College, Sialkot, Pakistan \\ ${ }^{3}$ Department of Surgery, Pak Red Crescent Medical and Dental College, Kasur, Pakistan \\ ${ }^{4}$ Department of Surgery, King Edward Medical University, Lahore, Pakistan
}

\begin{abstract}
Calcium oxalate phosphate is extremely rare composition of gall stones, with only one case reported in pediatric population till date. We report a case of pediatric cholelithiasis with a unique composition of calcium oxalate phosphate, detected at eight years of age. Its etiology remains unknown. An 8-year girl presented in Emergency Room with complaints of acute pain in right upper quadrant and nausea without any complaints of jaundice or fever. She was admitted to the hospital with the provisional diagnosis of acute cholecystitis keeping in view her symptoms and obesity, which was later found to be true after an ultrasound report; but the uniqueness and rarity of this case was determined after cholecystectomy, when the specimen containing her gall bladder along with stones was sent for analysis of the composition, which showed the rarest composition i.e., calcium oxalate phosphate. We report our experience on the unique composition of gallstones in a young girl with no known risk factors except obesity. These rare pediatric gallstones have not been associated with obesity in any literature earliar.
\end{abstract}

Key Words: Gallstones, Calcium oxalate phosphate, Pediatric.

How to cite this article: Liaqat A, Attiq H, Liaqat $\mathrm{H}$, Hassan L, Waheed R, Asghar T. Unique Pediatric Gallstones Composed of Calcium Oxalate Phosphate. J Coll Physicians Surg Pak 2020; 30(08):877-879.

\section{INTRODUCTION}

Although cholelithiasis is not a common condition in children, recent studies have documented an increasing incidence rate, owing to the development of better diagnostic tools. The prevalence of cholelithiasis in children has been reported to be $0.13 \%$ $0.3 \%$, whereas in obese children and adolescents, the prevalence rate has been estimated at $2 \%-6.1 \% .{ }^{1}$ Etiologically, cholelithiasis in children can be divided into three groups; hemolytic, other known etiology, and idiopathic. Almost $20 \%$ to $30 \%$ of all gallstones in children are due to hemolytic diseases such as sickle-cell disease, hereditary spherocytosis and thalassemia. In around $40 \%$ to $50 \%$ of cases, gallstones are due to another known etiology such as total parenteral nutrition, prolonged fasting, ileal disease or ileal resection, furosemide therapy, congenital biliary diseases such as choledochal cyst, chronic liver disease and progressive familial intrahepatic cholestasis (PFIC). Around $30 \%$ to $40 \%$ of cases are idiopathic. As in adults, gallstones in adolescent girls are more often idiopathic. ${ }^{2}$

Correspondence to: Dr. Ayesha Liaqat, Department of Surgery, Services Institute of Medical Sciences, Lahore, Pakistan

E-mail: ayeshaliaqat123@gmail.com

Received: March 11, 2019; Revised: November 04, 2019;

Accepted: November 14, 2019

DOI: https://doi.org/10.29271/jcpsp.2020.08.877
The etiology of gallstones with a composition of calcium oxalate phosphate is unknown and there are only numbered cases of such stones in literature.

\section{CASE REPORT}

An 8-year girl presented with acute pain in right upper quadrant and nausea. There was no complaint of vomiting, fever or jaundice. The patient was obese with weight of $65 \mathrm{kgs}$ (body mass index $40 \mathrm{~kg} / \mathrm{m}^{2}$. She had no comorbidities like diabetes or hypertension.

An ultrasound revealed distended gall bladder containing multiple calculi collectively measuring up to $40 \mathrm{~mm}$ with mild prominence of gallbladder and grade I hepatic steatosis. Common bile duct was not dilated. Liver parenchyma had mildly increased echogenicity, suggestive of fatty change. No focal hepatic mass lesion, intra-or extra-hepatic biliary dilatation was seen. Portal vein had normal diameter. Spleen, pancreas, urinary bladder and kidneys showed no abnormalities.

To rule out other differentials, her laboratory tests were sent that showed elevated erythrocyte sedimentation rate (ESR) of $54 \mathrm{~mm} / 1^{\text {st }} \mathrm{hr}$. (normal: $10 \mathrm{~mm} / \mathrm{hr}-20 \mathrm{~mm} / \mathrm{hr}$ ), decreased hematocrit of $39.3 \%$ (normal:42.00-47\%), mean corpuscular hemoglobin of $25.8 \mathrm{pg}$ (normal $29-32 \mathrm{pg}$ ) with normal hemoglobin level of $12.5 \mathrm{~g} / \mathrm{dL}$, normal white blood cell count and normal platelet count. Liver function tests revealed normal alkaline phosphatase level of $161 \mathrm{IU} / \mathrm{L}$ albumin $5.2 \mathrm{mg} / \mathrm{dL}$ (normal: 
3.5-5.4 mg/dL), total protein $9.6 \mathrm{~g} / \mathrm{dL}$ (normal: $6.30-8.30 \mathrm{~g} / \mathrm{dL}$ ); bilirubin (conjugatedand unconjugated), aspartateaminotransferase, alanine aminotransferase and gamma glutamyl transferase levels were normal. Enzyme-Linked immunosorbent assay results were non-reactive for anti-hepatitis $C$ virus antibodies and hepatitis B surface antigen. Serum calcium and parathyroid hormone levels were within normal range.

Final diagnosis of pediatric cholelithiasis was made and laparoscopic cholecystectomy was done.

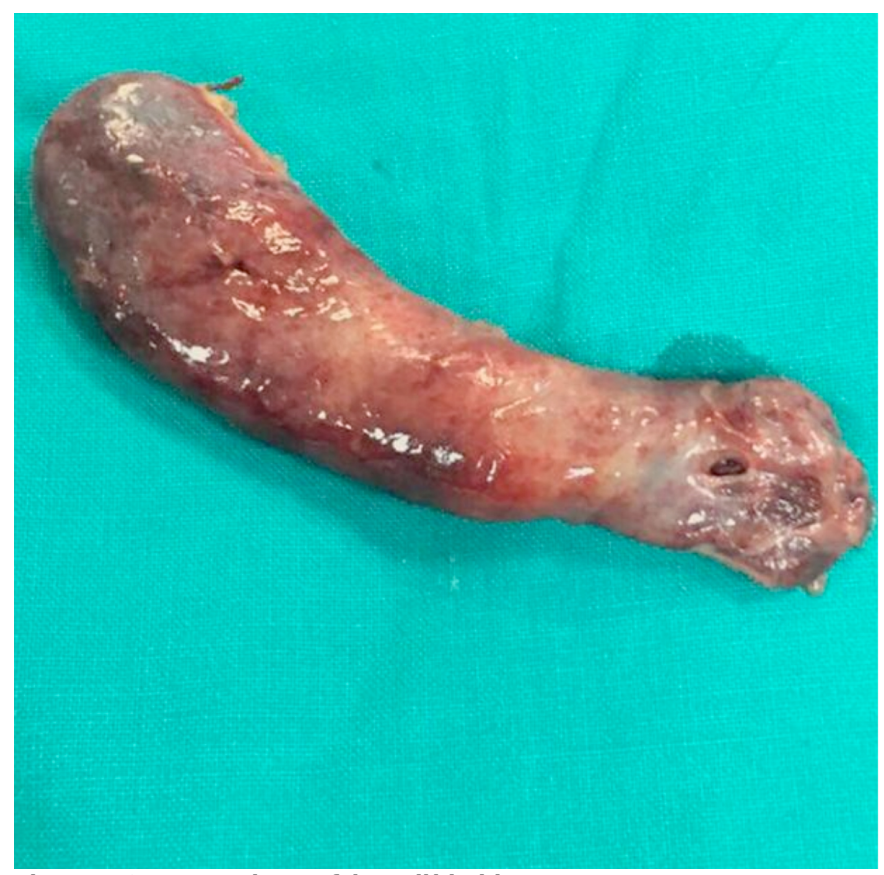

Figure 1: Gross specimen of the gall bladder.

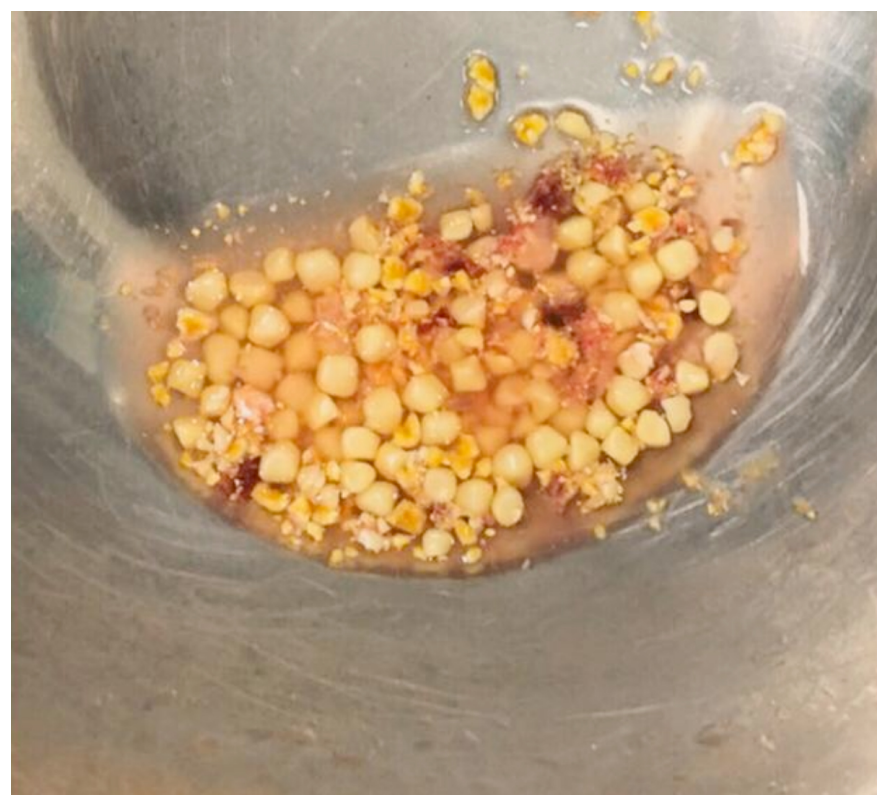

Figure 2: Grossspecimen of gallstones composed of calcium oxalate phosphate.

Gross specimen of gall bladder was examined, measuring $9 \times 3 \times 1 \mathrm{~cm}$. Mucosa was smooth and velvety. Thickness of wall measured $0.3 \mathrm{~cm}$. Based on histopathological examination, a diagnosis of chronic cholecystitis was made (Figure 1). On cutting open the gall bladder, multiple brown stones, each measuring $0.6 \times 0.4 \times 0.3 \mathrm{~cm}$, with irregular shapes were revealed (Figure 2). Chemical analysis showed the composition to be calcium $45 \%$, oxalate $35 \%$, phosphate $10 \%$ and uric acid $10 \%$. Patient is currently doing well on follow-up.

\section{DISCUSSION}

What makes this case unique is the presence of calcium oxalate phosphate gallstones in an obese child with no congenital or developmental abnormalities. The only case reported till date in literature was of a premature newborn with multiple congenital abnormalities including patent ductus arteriosus (PDA) and was placed on furosemide and given multiple treatments for acquired infections. ${ }^{3}$

The absence of any of the above risk factors and presence of obesity only makes this case one of its kind till date.

The cause of formation of oxalate gallstones is not known. Normally, the concentration of oxalate is not high in bile. This patient was not given oxalate in any form so as to cause elevated concentration of serum oxalate. After studying some reports in literature, we found that oxalate may be formed by fungi and that glyoxylate, its immediate precursor, is formed by bacteria but demonstration of oxalate formation by microorganisms in vivo has not been shown. ${ }^{4}$ Therefore, it may be assumed that the source of oxalate may be fungal or bacterial infection in this patient. Our diet may play some role in endogenous oxalate formation. Increased production of oxalate was found in patients who underwent jejunoileal bypass surgery. (This was not done in this patient, ruling it out as the risk factor for her gallstones.

As far as cholelithiasis in children is concerned, there is a studied change in the overall variety of this disease with worldwide increase in its incidence. Risk factors of childhood cholelithiasis include hemolytic diseases (20-30\%), other known etiology (40-50\%) such as fasting for longer periods, total parenteral nutrition, ileal disease / ileal resection, treatment with furosemide for longer times resulting in dehydration and abnormalities in ion concentrations, congenital biliary diseases such as choledochal cyst, chronic liver disease and PFIC. ${ }^{5}$ More than one-third of cases are idiopathic. Ultrasound is the most common diagnostic test with sensitivity and specificity exceeding $95 \%$. For diagnosing acute cholecystitis, cholescintigraphy with technetium $99 \mathrm{~m}$ labeled, is the most accurate method. ${ }^{6}$

Laparoscopic cholecystectomy is a safe and efficacious treatment for pediatric cholelithiasis with low rate of postoperative complications. $^{7}$

Less is known about the pathogenesis of childhood cholelithiasis and least is known about such a rare composition in pediatric population, hence guidelines for managementare lacking.

Cholelithiasis with such a unique chemical composition of 
calcium oxalate phosphate has never been reported without any family history or associated risk factors. All the cases of pediatric cholelithiasis should undergo chemical analysis and their etiologies need to be further studied as little information is present in literature. This step is important for their prevention and to decrease the rising incidence of pediatric cholelithiasis ingeneral.

\section{CONFLICT OF INTEREST:}

The authors declared no conflict of interest.

\section{PATIENT'S CONSENT:}

Informed consent was obtained from the patient's parents.

\section{AUTHORS' CONTRIBUTION:}

AL: Drafting the case report, analysis and interpretation. HA: Critically reviewed and analysed the data.

$\mathrm{HL}$ : Concept, design and retrieving data from previous related case reports.

LH: Drafting and analysis.

RW: Obtained patient's consent to publish the data and helped in retrieving the detailed history, examination, and critically analysing.

TA: Surgeon of the patient, analysed the patient data, gave final approval to the collected data and supervised the analysis and drafting.

\section{REFERENCES}

1. Karami H, Klanifer HR, Karami S. Cholilithiasis in children: A diagnostic and therapeutic approach. J Pediatric Rev 2017; 5 (1):e9114.

2. Podar U. Cholilithiasis in children. J Indian Pediatrics 2010; 47.

3. Callahan J, Hailer JO, Cacciarelli A. Cholelithiasis in infants: Association with total parenteral nutrition and furosemide. Radiology 1982; 143(2):437-9.

4. Wolf P, Mannino F, Hofmann AF, Nickoloff B, Edwards DK. Calcium oxalate phosphate gallstones: A unique chemical type of gallstones. J Clini Chem 1982; 28(8):1804-5.

5. Gallbladder gallstones (calculi). Available from: http:// www.ncbi.nlm.nih.gov/books/NBK459370/.

6. Gallbladder stones: Imaging and intervention. Available from:http://pubs.rsna.org/doi/10.1148/radiographics.20.3.g 00ma16751.

7. Laproscopic cholecystectomy in the pediatric population. Available from: http://www.liebertpub.com/doi/10.1089/ lap.2007.0150. 